University of Wollongong

Research Online

Faculty of Engineering and Information

Faculty of Engineering and Information

Sciences - Papers: Part A

Sciences

$1-1-2015$

Optical bistability induced by nonlinear surface plasmon polaritons in graphene in terahertz regime

\author{
Matthew Sanderson \\ University of Wollongong, ms919@uowmail.edu.au \\ Yee Sin Ang \\ University of Wollongong, ysa190@uowmail.edu.au \\ Sen Gong \\ University of Electronic Science and Technology of China \\ Tao Zhao \\ University of Electronic Science and Technology of China \\ Min $\mathrm{Hu}$ \\ University of Electronic Science and Technology of China
}

See next page for additional authors

Follow this and additional works at: https://ro.uow.edu.au/eispapers

Part of the Engineering Commons, and the Science and Technology Studies Commons

Research Online is the open access institutional repository for the University of Wollongong. For further information contact the UOW Library: research-pubs@uow.edu.au 


\title{
Optical bistability induced by nonlinear surface plasmon polaritons in graphene in terahertz regime
}

\author{
Abstract \\ We demonstrate optical bistability in a prism-air-graphene-dielectric structure. Under a moderate electric \\ field in the terahertz frequency regime, the third order nonlinear optical conductivity is comparable to the \\ linear conductivity. The nonlinear conductivity enhances the energy of surface plasmon polaritons. Both \\ the energy and frequency of the surface plasmon polaritons depend on the strength of the nonlinear \\ current in the graphene layer. When considering excitation in the Kretschmann configuration, the \\ reflectance as a function of frequency exhibits bistability. The origin of the bistability is the field \\ dependence of the plasmon mode. We have determined the parameter regime for the occurrence of \\ bistability in this structure.

\section{Disciplines} \\ Engineering | Science and Technology Studies

\section{Publication Details} \\ Sanderson, M., Ang, Y., Gong, S., Zhao, T., Hu, M., Zhong, R., Chen, X., Zhang, P., Zhang, C. \& Liu, S. (2015). \\ Optical bistability induced by nonlinear surface plasmon polaritons in graphene in terahertz regime. \\ Applied Physics Letters, 107 (20), 203113-1-203113-4.

\section{Authors} \\ Matthew Sanderson, Yee Sin Ang, Sen Gong, Tao Zhao, Min Hu, Renbin Zhong, Xiaoxing Chen, Ping Zhang, \\ Chao Zhang, and Shenggang Liu
}




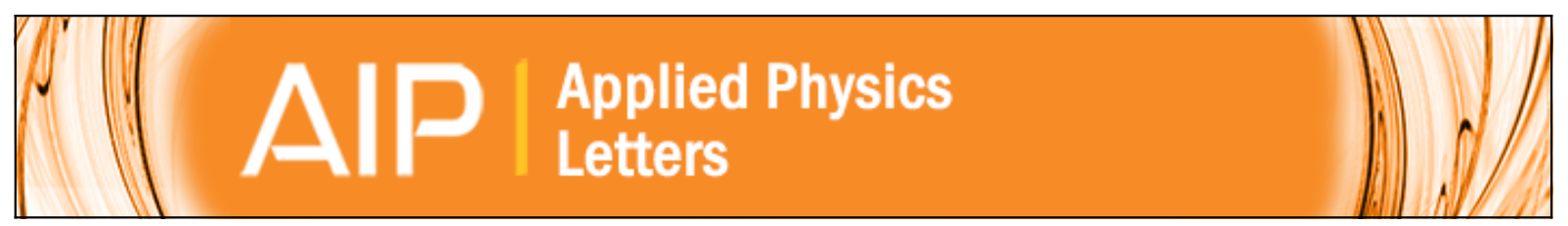

\section{Optical bistability induced by nonlinear surface plasmon polaritons in graphene in} terahertz regime

Matthew Sanderson, Yee Sin Ang, Sen Gong, Tao Zhao, Min Hu, Renbin Zhong, Xiaoxing Chen, Ping Zhang,

Chao Zhang, and Shenggang Liu

Citation: Applied Physics Letters 107, 203113 (2015); doi: 10.1063/1.4936232

View online: http://dx.doi.org/10.1063/1.4936232

View Table of Contents: http://scitation.aip.org/content/aip/journal/apl/107/20?ver=pdfcov

Published by the AIP Publishing

\section{Articles you may be interested in}

Transformation of surface plasmon polaritons to radiation in graphene in terahertz regime

Appl. Phys. Lett. 106, 223107 (2015); 10.1063/1.4922261

Extremely confined terahertz surface plasmon-polaritons in graphene-metal structures

Appl. Phys. Lett. 103, 071103 (2013); 10.1063/1.4818660

Efficient terahertz electro-absorption modulation employing graphene plasmonic structures

Appl. Phys. Lett. 101, 261115 (2012); 10.1063/1.4773374

Nonlinear optical conductance in a graphene pn junction in the terahertz regime

Appl. Phys. Lett. 97, 011907 (2010); 10.1063/1.3462972

Optical bistability enhanced by highly localized bulk plasmon polariton modes in subwavelength metal-nonlinear dielectric multilayer structure

Appl. Phys. Lett. 94, 081117 (2009); 10.1063/1.3079408

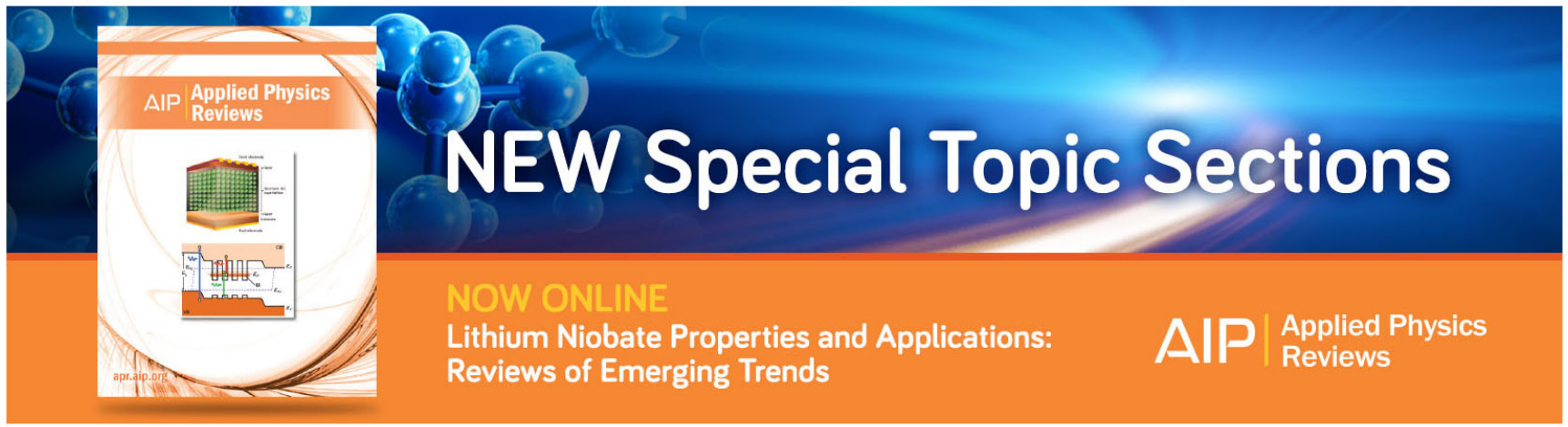




\title{
Optical bistability induced by nonlinear surface plasmon polaritons in graphene in terahertz regime
}

\author{
Matthew Sanderson, ${ }^{1}$ Yee Sin Ang, ${ }^{1}$ Sen Gong, ${ }^{2,3}$ Tao Zhao, ${ }^{2,3}$ Min Hu, ${ }^{2,3}$ Renbin Zhong, ${ }^{2,3}$ \\ Xiaoxing Chen, ${ }^{2,3}$ Ping Zhang, ${ }^{2,3}$ Chao Zhang, ${ }^{1,3, a)}$ and Shenggang Liu ${ }^{2,3}$ \\ ${ }^{1}$ School of Physics, University of Wollongong, New South Wales 2522, Australia \\ ${ }^{2}$ Terahertz Research Center, School of Physical Electronics, University of Electronic Science and Technology \\ of China, Chengdu, Sichuan 610054, China \\ ${ }^{3}$ Cooperative Innovation Centre of Terahertz Science, Chengdu, Sichuan 610054, China
}

(Received 28 July 2015; accepted 10 November 2015; published online 20 November 2015)

\begin{abstract}
We demonstrate optical bistability in a prism-air-graphene-dielectric structure. Under a moderate electric field in the terahertz frequency regime, the third order nonlinear optical conductivity is comparable to the linear conductivity. The nonlinear conductivity enhances the energy of surface plasmon polaritons. Both the energy and frequency of the surface plasmon polaritons depend on the strength of the nonlinear current in the graphene layer. When considering excitation in the Kretschmann configuration, the reflectance as a function of frequency exhibits bistability. The origin of the bistability is the field dependence of the plasmon mode. We have determined the parameter regime for the occurrence of bistability in this structure. (C) 2015 AIP Publishing LLC.

[http://dx.doi.org/10.1063/1.4936232]
\end{abstract}

In a nonlinear system, due to a self feedback mechanism, multiple stable states under a given external condition can occur. For example, in a two dimensional semiconductor double-barrier-resonant-tunneling (DBRT) junction, the tunneling I-V curve exhibits tristability when the tunneling is assisted by the plasmon excitation in the resonant quantum well. ${ }^{1}$ In the DBRT junction, the tunneling current is determined by the excitation in the resonant well where the plasmon excitation occurs, and the plasmon energy is determined by the charge concentration or the current. As a result, there is a selfconsistent condition to determine the current and tristability occurs in the I-V characteristics. In practice, only the top and bottom states can be probed experimentally and as a result the phenomenon is more often referred to as bistability. Such a transport bistability can also occur when there is a nonlinear electron-photon coupling in the resonant well. ${ }^{2}$ Optical bistability occurs when light is reflected by a metal surface where surface plasmon polaritons (SPPs) are excited. ${ }^{3}$ With recent rapid progress in graphene research, many different avenues to explore nonlinear effects have opened up. Graphene has shown exceptional electronic, mechanical, and optical properties, ${ }^{4-8}$ such as very high electronic mobility and universal conductance as well as the carrier concentration and the plasmon frequency in graphene exhibiting tunability by varying the gate voltage. Graphene based radiation sources, detectors, and modulators ${ }^{9-14}$ have been proposed and investigated and it has been shown that graphene is a strong nonlinear material which could lead to many more potential applications. ${ }^{15-20}$ The required threshold field for graphene to exhibit the nonlinear effect is rather moderate, in the order of a few $\mathrm{kV} / \mathrm{cm}$.

Recent experimental and theoretical works have shown that graphene can support SPP's. ${ }^{21-27}$ There is a significant enhancement (up to two orders of magnitude) of light radiation from graphene SPPs where the graphene sheet is deposited on a periodic dielectric substrate. We have shown recently that the strong nonlinear conductivity in graphene can lift the SPP dispersion above the photon line in a dielectric. ${ }^{28}$ As a result, the SPPs can be transformed into radiation by a moving electron beam. When light is incident on the dielectrics above the graphene and reflected at the interface, the SPPs can be excited. In the nonlinear regime, the SPP energy is dependent on the intensity of the incident light and the reflection is dependent on the SPP energy. This gives rise to the self-consistent condition for the reflection coefficient. We shall demonstrate that the reflection coefficient involving nonlinear SPPs in graphene can give rise to optical bistability. Optical bistability can also occur when we consider one of the dielectric layers to be nonlinear; ${ }^{3}$ however, graphene is a stronger nonlinear material and nonlinearity occurs in graphene at a weaker field under which the dielectrics remain a linear system. There has recently been some theoretical interest in optical bistability involving the nonlinear effect of graphene in a 4-layer structure, ${ }^{29}$ and in a free-standing graphene sheet. ${ }^{30}$ The result presented in this work is quite distinct to these previous works. In Ref. 29, only the linear conductivity is complex while the nonlinear conductivity is purely imaginary. As a result, there were no nonlinear SPPs being excited on the graphene sheet. In Ref. 30, SPPs were not present at all since it is free standing graphene. The origin of the bistability reported here is due to the nonlinear SPPs. Therefore, the bistability characteristics in both the frequency domain and the field domain in the present system are also quite different to those of previous systems.

In our analysis, we shall only consider a situation where the frequency of the incident field is low such that only the intraband transition in graphene is involved. The intraband conductivity can be obtained by solving the Boltzmann equation ${ }^{31}$

$$
\frac{\partial f}{\partial t}-\frac{\partial f}{\partial \mathbf{p}} \cdot \mathbf{E}=-\frac{f-f_{0}}{\tau},
$$


where $f_{0}=\left[1+\exp \left(\frac{\varepsilon-\mu}{k_{B} T}\right)\right]^{-1}$ is the equilibrium Fermi-Dirac distribution, $\varepsilon=v_{F}|\mathbf{p}|$ is the linear energy dispersion for a graphene sheet, $\mathbf{p}=p(\cos \varphi, \sin \varphi)=\left(p_{x}, p_{y}\right)$ is the momentum, $v_{F}$ is the Fermi velocity, $f$ is the non-equilibrium Fermi-Dirac distribution, $\mu$ is the chemical potential, and $\tau$ is the relaxation time of electrons and holes in the graphene sheet. As we need to include charge carriers in the lower band (where $\epsilon=-v_{F}|\mathbf{p}|$ ), we shall henceforth use the notation that $f=f_{+}-f_{-}$, where the $+(-)$refer to the upper and lower bands, respectively.

To find the non-linear distribution function, $f$, we expand it into components oscillating at frequencies from $-n \omega$ to $n \omega$. The nth order nonlinear term (the term proportional to the $\pm n \omega$ power of the electric field) can be written as

$$
f_{n}=\sum_{m=0}^{n} f_{m}(t)=\sum_{m=0}^{n} \sum_{k=-m}^{m} f_{m}^{k \omega} \mathrm{e}^{i k \omega t}
$$

If we substitute this into the Boltzmann transport equation and match terms with the same frequency when using an electric field along the x-axis, $\mathbf{E}=\frac{E_{0}}{2} \hat{x}\left(\mathrm{e}^{i \omega t}+\mathrm{e}^{-i \omega t}\right)$, we obtain

$f_{n}^{ \pm(n-2 l) \omega}=\frac{e E_{0}}{2\left[\tau^{-1} \pm i(n-2 l) \omega\right]}\left[\frac{\partial f_{n-1}^{ \pm(n-2 l-1) \omega}}{\partial p_{x}}+\frac{\partial f_{n-1}^{ \pm(n-2 l+1) \omega}}{\partial p_{x}}\right]$

for $0 \leq l \leq\left\lfloor\frac{n}{2}\right\rfloor$, where $E_{0}$ is the incident field strength. For the $n$th order term oscillating at frequency $n \omega$, we have the simpler form

$$
f_{n}^{ \pm n \omega}=\frac{e E_{0}}{2\left(\tau^{-1} \pm i n \omega\right)} \frac{\partial f_{n-1}^{ \pm(n-1) \omega}}{\partial p_{x}} .
$$

The nth order conductivity at frequency $k \omega$ can be written as

$$
J_{n}^{\text {intra }}(k \omega)=-\frac{1}{\pi^{2} \hbar^{2}} \sum_{ \pm} \int( \pm e) v_{x} f_{n, \pm}^{k \omega} \mathrm{d} \mathbf{p},
$$

where the summation over the two bands will be suppressed from here on. It is noted that only the terms with odd $n$ contribute to the nonlinear current. Terms with even $n$ vanish after integration over the momentum space.

The $n$th order current is of the form $J=\sum_{i=0}^{n} \sigma_{i} E^{i}$. By comparing terms oscillating at the same frequency, we can find the conductivity at any frequency and order. From Eqs. (2)-(5), the linear and lowest order nonlinear conductivities are given as 28,30

$$
\begin{gathered}
\sigma_{1}(\xi)=-\frac{4}{\pi} \sigma_{0} \frac{1}{\gamma-i \xi} \mathcal{I}_{1}, \\
\sigma_{3}(\xi)=-\frac{12}{\pi} \sigma_{0} \phi^{2} \frac{1}{\left(\gamma^{2}+\xi^{2}\right)(\gamma-2 i \xi)} \mathcal{I}_{3}, \\
\sigma_{3}(3 \xi)=-\frac{4}{\pi} \sigma_{0} \phi^{2} \frac{1}{(\gamma-i \xi)(\gamma-2 i \xi)(\gamma-3 i \xi)} \mathcal{I}_{3} .
\end{gathered}
$$

Here $p, \omega$, and $\tau^{-1}$ are all given in units of energy, i.e., $v_{F} p$ $=\varepsilon \rightarrow p, \hbar \omega \rightarrow \xi$, and $\hbar \tau^{-1} \rightarrow \gamma$. Also, $\sigma_{0}=\frac{e^{2}}{4 \hbar}, \phi=\hbar e v_{F}$ $=6.582119 \times 10^{-8} \mathrm{eV}^{2} \mathrm{~cm} / \mathrm{V}$ and $E_{0}$, the incident field, is specified in units of $\mathrm{V} / \mathrm{cm}$. The $\mathcal{I}_{n}$ terms in Eqs. (6)-(8) are given by

$$
\mathcal{I}_{n}=\frac{1}{\pi} \int_{0}^{\infty} \int_{0}^{2 \pi} \frac{\partial^{n} f_{0}}{\partial p_{x}^{n}} \cos \varphi \mathrm{d} \varphi p \mathrm{~d} p
$$

At $T \neq 0$ we can retrieve the expected ${ }^{32} 1$ st order conductivity

$$
\sigma_{1}^{i n t r a}=\frac{4 \sigma_{0}}{\pi} \frac{1}{\gamma-i \xi}\left\{\mu+2 k_{B} T \ln \left[1+\mathrm{e}^{-\mu / k_{B} T}\right]\right\} .
$$

It is possible to calculate the values of $\mathcal{I}_{n}$ analytically when $T=0$ for any $n$. 1st and 3 rd orders at $T=0$ are given by $\mathcal{I}_{1}=-\mu$ and $\mathcal{I}_{3}=\frac{3}{4} \frac{1}{\mu}$, respectively. As has been seen previously, ${ }^{32}$ SPPs can be efficiently excited in the Kretchmann configuration. We now show that the optical nonlinearity of the graphene layer will result in optical bistability when attempting to excite SPPs with a strong incident field.

The model system is shown schematically in Fig. 1. A graphene layer is grown on a dielectric with dielectric constant $\epsilon_{1}$ and above the graphene sheet is a different dielectric with dielectric constant $\epsilon_{2}$. The system we are considering is shown in Fig. 1(b). In this case, a prism $\left(\epsilon_{3}\right)$ is placed at distance $d$ above the air layer $\left(\epsilon_{2}\right)$. This configuration will allow for the SPPs to be excited provided the incident angle is such that the wavevector of the incident photons match those of the SPPs. The field components are determined by the boundary conditions. At $z=0$ (the boundary between $\epsilon_{3}$ and $\epsilon_{2}$ )

$$
\begin{aligned}
& E_{x}^{i}+E_{x}^{r}=E_{2, x}^{+}+E_{2, x}^{-}, \\
& B_{y}^{i}+B_{y}^{r}=B_{2, y}^{+}+B_{2, y}^{-} .
\end{aligned}
$$

At $z=d$ (the boundary between $\epsilon_{2}$ and $\epsilon_{1}$ )

$$
\begin{gathered}
E_{1, x}^{-} \mathrm{e}^{-\kappa_{1} d}=E_{2, x}^{+} \mathrm{e}^{\kappa_{2} d}+E_{2, x}^{-} \mathrm{e}^{-\kappa_{2} d}, \\
B_{1, y}^{-} \mathrm{e}^{-\kappa_{1} d}=B_{2, y}^{+} \mathrm{e}^{\kappa_{2} d}+B_{2, y}^{-} \mathrm{e}^{-\kappa_{2} d} \\
-\mu_{0}\left[\sigma_{1}+\sigma_{3}\left|E_{1, x}^{-}\right|^{2} \mathrm{e}^{-2 \kappa_{1} d}\right] E_{1, x}^{-} \mathrm{e}^{-\kappa_{1} d} .
\end{gathered}
$$

From Eqs. (11) and (12), we obtain the following nonlinear relationship for $r=E_{r} / E_{i}$, the ratio of reflected to incident fields

$$
\begin{aligned}
& \frac{\epsilon_{2}}{\kappa_{2}}\left(\lambda_{-}+r \lambda_{+}\right)+\frac{\epsilon_{1}}{\kappa_{1}}\left(\Lambda_{-}+r \Lambda_{+}\right)-4 i \alpha \\
& \quad \times\left[\sigma_{1}(\xi)+\sigma_{3}(\xi) E_{i}^{2}\left|\Lambda_{-}+r \Lambda_{+}\right|^{2}\right]\left(\Lambda_{-}+r \Lambda_{+}\right)=0 .
\end{aligned}
$$

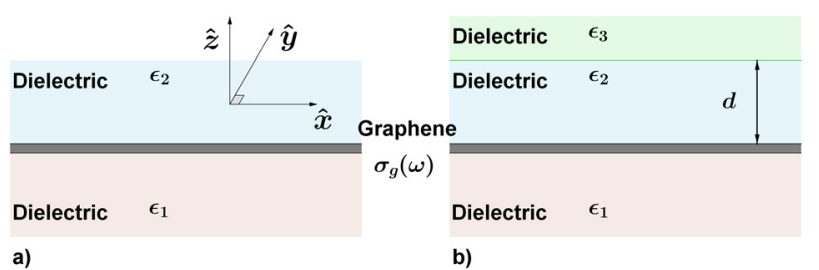

FIG. 1. The structures considered for calculating SPP dispersion relations. (a) A layer of graphene is sandwiched between two dielectrics. This is the usual setup for determining the dispersion relation of SPPs. (b) An extra dielectric layer is placed on top of $\epsilon_{2}$ (Kretschmann configuration). 


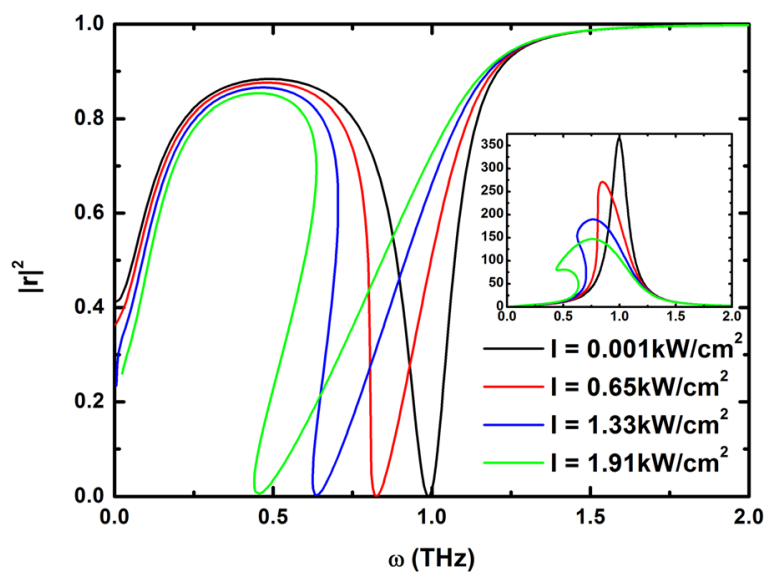

FIG. 2. Relative intensity of the reflected field. Inset shows the effective field enhancement on the lower side of the graphene sheet.

Here $\lambda_{ \pm}=\sinh \left(\xi \kappa_{2} d\right) \pm i \frac{\kappa_{2}}{k_{z}} \frac{\epsilon_{3}}{\epsilon_{2}} \cosh \left(\xi \kappa_{2} d\right), \Lambda_{ \pm}=\cosh \left(\xi \kappa_{2} d\right)$ $\pm i \frac{\kappa_{2}}{k_{z}} \epsilon_{3} \sinh \left(\xi \kappa_{2} d\right), \kappa_{j}=\sqrt{\epsilon_{3} \sin ^{2}(\theta)-\epsilon_{j}}$ and $k_{z}=\sqrt{\epsilon_{3}} \cos (\theta)$. $\theta$ is the angle of the incident light relative to the normal of $\epsilon_{3}$. The value for $t=E_{1, x}^{-} / E_{i}$ can be obtained from the relation $t \mathrm{e}^{-\kappa_{1} d}=\Lambda_{-}+r \Lambda_{+}$. At high fields, Eq. (13) gains an extra two complex roots. As a result, optical bistability occurs as can be seen in Fig. 2. In Figs. 2-5, we have used the following parameters: $\epsilon_{1}=5, \epsilon_{2}=1, \epsilon_{3}=14, \mu=0.45 \mathrm{eV}$, $\gamma=0.1 \mathrm{meV}$, and $d=2.51 \mu \mathrm{m}$ (unless specified in the plot). The incident intensity is defined as $I=\frac{3 c \epsilon_{0}}{2}|E|^{2} \approx 0.003981$ $|E|^{2} \mathrm{~W} / \mathrm{cm}^{2}$. The optical bistability has the nature of a feedback effect due to the nonlinear SPP's in the graphene layer. The nonlinear part of the optical conductivity is proportional to the electric field intensity (or the energy density of the electric field) and inversely proportional to the electronic energy. While the nonlinear conductivity does not seem to be large, the effect it has is still quite drastic due to the effective field enhancement on the lower side of the graphene sheet as seen in the inset of Fig. 2. For a specific angle and frequency, light reflecting through the prism will couple to the SPPs in the graphene sheet. If the light intensity is strong enough, the excited SPPs are nonlinear and the SPP energy is dependent on the amplitude and frequency of the incident light. Since the reflection can be viewed as a re-emission of

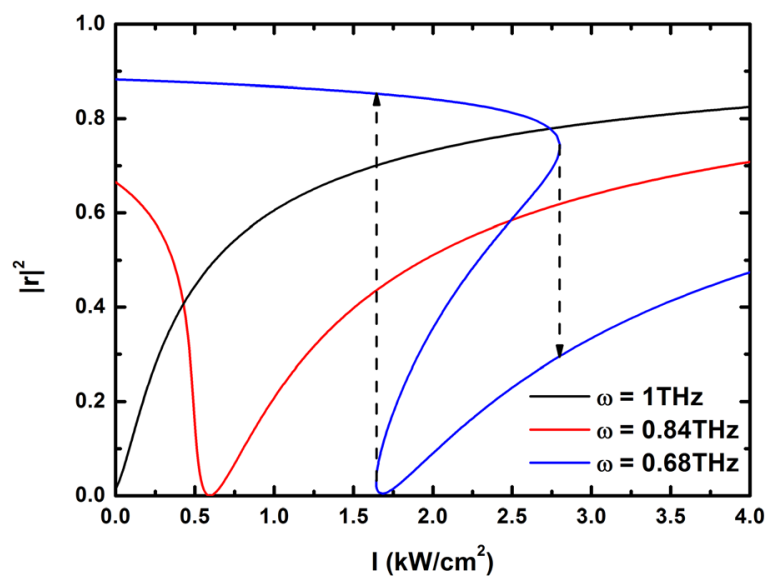

FIG. 3. Reflectivity at fixed frequencies. A hysteresis-like loop appears when we have bistability. light from the interface, the reflected light is dependent on the SPP energy. As a result, the reflectivity exhibits bistability. An interesting and unique property of the bistability reported here is that it can be tuned by varying the chemical potential of the graphene and the intensity and frequency of the incident light. The bistability in the frequency domain gives rise to a hysteresis loop when the reflected field is plotted as a function of the incident field as shown in Fig. 3. This hysteresis-like loop appears when we have an incident field with frequency above the critical frequency, $\omega_{c}$. This plot can be seen as vertical slices of Fig. 2 at different frequencies and with the field allowed to vary.

It should be noted that excitation of SPPs in graphene only occurs for a specific value of the incident angle, denoted $\theta_{0}$, which is a function of $d, \mu$, and $\gamma$. For any combination of these parameters, we can (sometimes) find a frequency, $\omega_{0}$, and angle, $\theta_{0}$, at which the reflectivity will be 0 in the linear case. The critical intensity $I_{c}$ and the critical frequency $\omega_{c}$ (the intensity and frequency at which at which an onset of bistability occurs) are plotted in Fig. 4.

It can be seen that there is a reasonably quadratic relationship between $I_{c}$ and $\mu$ and that $\omega_{c}$ decreases with $\mu$. Because of these two factors, we find that there is a chemical potential such that $I_{c}$ is minimised $\left(\sim 575 \mathrm{~W} / \mathrm{cm}^{2}\right.$ at $\sim 0.4 \mathrm{eV}$ in this case) and that there will be a cutoff value for $\mu$ below which bistability will no longer be possible due to the critical frequency being too close to 0 . Due to the complexity of the expressions, it is not possible to find an analytical relationship between $I_{c}, \omega_{c}$, and $\mu$.

The reflection characteristics (location and width) are strongly dependent on the relaxation rate $\gamma$. If $\gamma$ is too large a

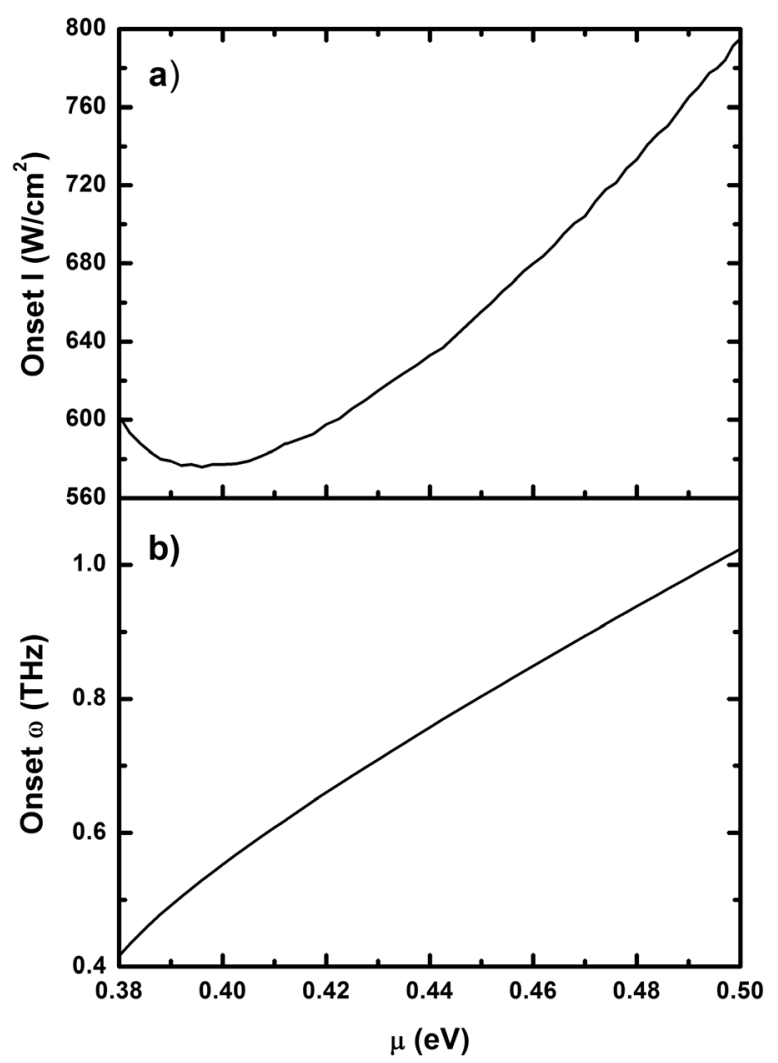

FIG. 4. (a) Minimum incident field intensity required to cause bistability. (b) Frequency at which bistability occurs at minimum field. 


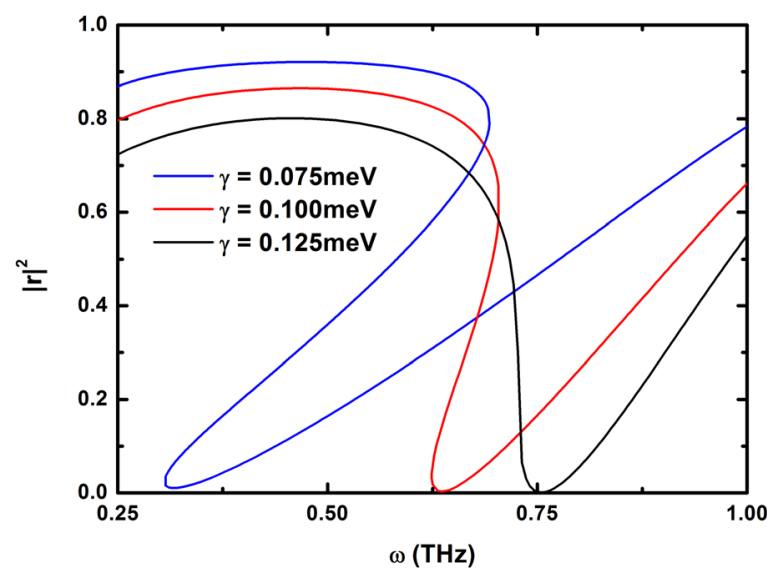

FIG. 5. Effect of the relaxation rate on the existence of bistability.

true zero in the reflection disappears. Fig. 5 shows the variation of bistability with $\gamma$. As $\gamma$ increases, the bistability is "straightened out," indicating that a larger field is required to observe bistability. The value of $d$ also has a similar effect. Both $d$ and $\gamma$ affect how the incident waves are being reflected (in the linear case), which in turn will affect the location and possibility of bistability. Finally, it should be pointed out that the nonlinear conductivity used in this has its limitations. As the field intensity increases further or the frequency decreases, the subsequent higher order terms in the conductivity can become important.

In summary, we have shown that the reflection of light at the interface of two dielectrics situated above a graphene sheet exhibits bistability which is caused by the excitation of nonlinear surface plasmon polaritons in the graphene layer.

This work was supported by the Australian Research Council Discovery Grant (No. DP140101501), and by the Chinese National Basic Research Program under Grant No. 2014 CB339801.

${ }^{1}$ C. Zhang, A. D. Martin, M. Lerch, P. E. Simmond, and L. Eaves, Phys. Rev. Lett. 72, 3397 (1994).

${ }^{2}$ C. Zhang, Appl. Phys. Lett. 78, 4187 (2001).

${ }^{3}$ C. Wu, G. Song, H. Liu, L. Cui, L. Yu, and J. Xiao, J. Mod. Opt. 60, 190 (2013).

${ }^{4}$ A. H. C. Neto, F. Guinea, N. M. R. Peres, K. S. Novoselovm, and A. K. Geim, Rev. Mod. Phys. 81, 109 (2009).
${ }^{5}$ K. S. Novoselov, A. K. Geim, S. Morozov, D. Jiang, M. Katsnelson, I. Grigorieva, S. Dubonos, and A. Firsov, Nature 438, 197 (2005).

${ }^{6}$ J. Horng, C.-F. Chen, B. Geng, C. Girit, Y. Zhang, Z. Hao, H. A. Bechtel, M. Martin, A. Zettl, M. F. Crommie, Y. Ron Shen, and F. Wang, Phys. Rev. B 83, 165113 (2011).

${ }^{7}$ Z. Fei, A. S. Rodin, W. Gannett, S. Dai, W. Regan, M. Wagner, M. K. Liu, A. S. McLeod, G. Dominguez, M. Thiemens, A. H. Castro Neto, F. Keilmann, A. Zettl, R. Hillenbrand, M. M. Fogler, and D. N. Basov, Nat. Nanotechnol. 8, 821-825 (2013).

${ }^{8}$ Z. Q. Li, E. A. Henriksen, Z. Jiang, Z. Hao, M. C. Martin, P. Kim, H. L. Stormer, and D. N. Basov, Nat. Phys. 4, 532-535 (2008).

${ }^{9}$ M. Liu, X. Yin, E. Ulin-Avila, B. Geng, T. Zentgraf, L. Ju, F. Wang, and X. Zhang, Nature 474, 64 (2011).

${ }^{10}$ A. K. Geim, Science 324, 1530 (2009).

${ }^{11}$ L. Ju, B. Geng, J. Horng, C. Girit, M. Martin, Z. Hao, H. A. Bechtel, X. Liang, A. Zettl, Y. Ron Shen, and F. Wang, Nat. Nanotechnol. 6, 630 (2011).

${ }^{12}$ M. Jablan, H. Buljan, and M. Soljacic, Phys. Rev. B 80, 245435 (2009).

${ }^{13}$ A. Vakil and N. Engheta, Science 332, 1291 (2011).

${ }^{14}$ F. Bonaccorso, Z. Sun, T. Hasan, and A. C. Ferrari, Nat. Photonics 4, 611 (2010).

${ }^{15}$ S. A. Mikhailov and K. Ziegler, J. Phys.: Condens. Matter 20, 384204 (2008).

${ }^{16}$ A. R. Wright, X. G. Xu, J. C. Cao, and C. Zhang, Appl. Phys. Lett. 95, 072101 (2009).

${ }^{17}$ X. G. Xu, S. Sultan, C. Zhang, and J. C. Cao, Appl. Phys. Lett. 97, 011907 (2010).

${ }^{18}$ J. Wang, Y. Hernandez, M. Lotya, J. N. Coleman, and W. J. Blau, Adv. Mater. 21, 2430 (2009).

${ }^{19}$ E. Hendry, P. J. Hale, J. Moger, A. K. Savchenko, and S. A. Mikhailov, Phys. Rev. Lett. 105, 097401 (2010).

${ }^{20}$ K. Yang, S. Arezoomandan, and B. Sensale-Rodriguez, Terahertz Sci. Technol. 6(4), 223 (2013).

${ }^{21}$ F. Rana, IEEE Trans. Nanotechnol. 7, 91 (2008).

${ }^{22}$ B. Wang, X. Zhang, X. Yuan, and J. Teng, Appl. Phys. Lett. 100, 131111 (2012).

${ }^{23}$ B. Wang, X. Zhang, F. J. Garcia-Vidal, X. Yuan, and J. Teng, Phys. Rev. Lett. 109, 073901 (2012).

${ }^{24}$ M. Farhat, S. Guenneau, and H. Bagci, Phys. Rev. Lett. 111, 237404 (2013).

${ }^{25}$ S. Liu, C. Zhang, M. Hu, X. Chen, P. Zhang, S. Gong, T. Zhao, and R. Zhong, Appl. Phys. Lett. 104, 201104 (2014).

${ }^{26}$ T. Zhan, D. Han, X. Hu, X. Liu, S.-T. Chui, and J. Zi, Phys. Rev. B 89, 245434 (2014).

${ }^{27}$ S. Liu, M. Hu, R. Zhong, X. Chen, P. Zhang, S. Gong, and T. Zhao, Terahertz Sci. Technol. 8(2), 69 (2015).

${ }^{28}$ S. Gong, T. Zhao, M. Sanderson, M. Hu, X. Chen, P. Zhang, R. Zhong, C. Zhang, and S. G. Liu, Appl. Phys. Lett. 106, 223107 (2015).

${ }^{29}$ X. Dai, L. Jiang, and Y. Xiang, Opt. Express 23, 6497 (2015).

${ }^{30}$ N. M. R. Peres, Y. V. Bludov, J. E. Santos, A. Jauho, and M. I. Vasilevskiy, Phys. Rev. B 90, 125425 (2014).

${ }^{31}$ C. Kittel, Introduction to Solid State Physics, 8 th ed. (Wiley, 2005).

${ }^{32}$ Yu. V. Bludov, A. Ferreira, N. M. R. Peres, and M. I. Vasilevskiy, Int. J. Mod. Phys. B 27, 1341001 (2013). 